\title{
EDITORIAL
}

\section{DENGUE, WHAT WE LEARNT FROM THE RECENT TRENDS}

When a sero survey on dengue conducted at Chattogram city in late 1990s found around 13\% cases of acute febrile illness positive for dengue it was a surprise. ${ }^{1}$ Following first significant outbreak in 2000, dengue is now recognized as a major public health problem. There was a rapid global spread of dengue disease. To note some salient aspects of dengue: absence of simple diagnostic tool for the early phase, absence of specific therapy, and absence of effective \& efficient vector control. Dengue is now also considered as a climate sensitive emerging neglected tropical disease, related with rainfall, temperature, humidity; breeding places and time of incubation period has now found to be correlated with temperature. A similar phenomenon happened with Vibrio Cholerae in the past and with sudden upsurge of cases of malaria in the Chattogram Hill Districts in $2014 .^{2}$

A brief revisit of program on vector borne diseasesmalaria program amalgamated initially with primary health care, later with CDC, DGHS was renamed as vector borne diseases cinbrel (VBDC) program, and recently named as Malaria \& aedes transmitted diseases unit DGHS. During outbreak the Emergency Cell of DGHS, Government of Bangladesh take care of reporting of selected illness including dengue on daily basis based on compilation of cases, although few hospitals reporting, recently increased number of hospitals are found to be reporting.

Infectious diseases Act 2019 has been promulgamated with 23 diseases to be reported by health care professionals which is yet to be operationalized.

There was a recent survey on different entomological indices in Dhaka city and all over which gave a warning of possible increase of cases in 2019.

In 2000- in Bangladesh a total of $\sim 5000$ cases of dengue, with $<100$ deaths were repported. In 2019 cases increased to $\sim 100,000$ with little more than $\sim 120$ official confirmed death (Figure: 1).

Bangladesh J Medicine 2020; 31 : 1-2

DOI: https://doi.org/10.3329/bjmed.v31i1.44745

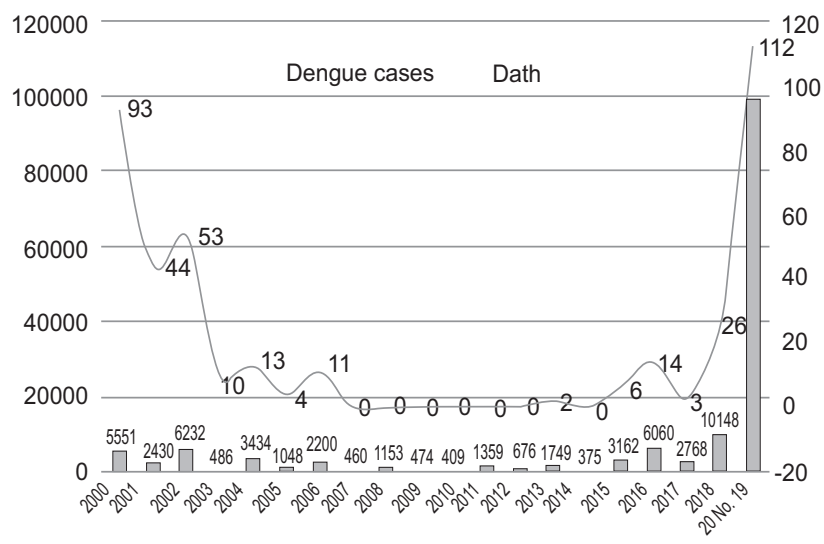

Fig.-1: Reported Dengue Cases and Deaths, Bangladesh: $2000-2019$

Even in the last week of November, December there are reports of cases of dengue being admitted in different hospitals (Figure: 2). ${ }^{3}$

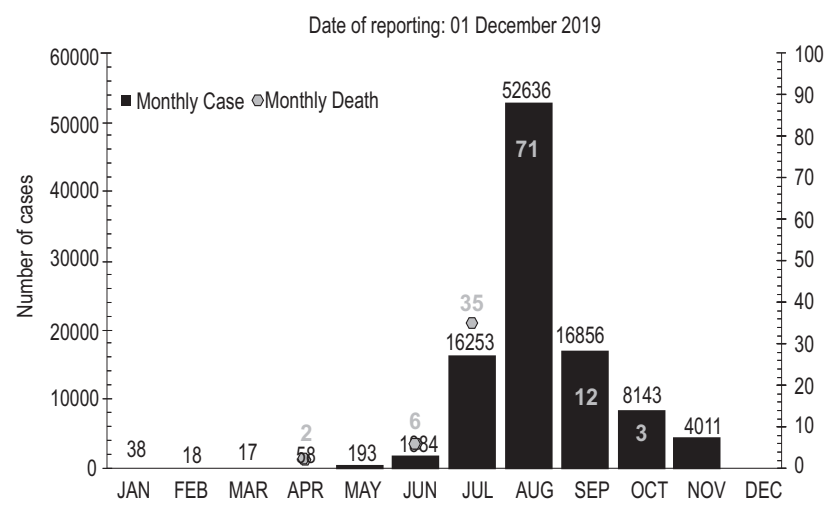

Fig.-2: Health Emergency Operation Center and Control Room, DGHS: Reported Monthly Dengue \& suspected Dengue Cases Confirmed Deaths, Bangladesh.

The number of cases of dengue in 2019 is likely to be under reported, the actual number may be many folds higher. ${ }^{4}$

Dengue become an endemic in Bangladesh with spread to even rural township.

During the outbreak in 2000 expectations was, to do haematocrit for assessing the cases, providing fluid 
management and heavily reliant mostly on platelet transfusion with a hue and cry for cell separator. In 2019 NS1 has been found to be used during acute phase, and IgG, IGM to see the status of infection, and the professionals and the community aspiring to use more ICU care for management with a large number of media reports of death. Triage was tried as well in hospitals flooded with cases to identify severe cases. While providing care- management focused at ICU based in some occasion, fluid management at ICU and even in some hospitals dedicated unit for dengue was established. There was also lack of proper training initially of ICU physicians.

During early part of outbreak general wards were used for management later on dengue dedicated ward was established. In Bangladesh there are a large variety of specialists but infectious diseases is not considered as specialty. The quick setting up of dedicated dengue ward gave some confidence among the public and the professionals. Specific dedicated ID ward is now more urgent to consider in medical college hospitals and in medical universities which will be able to cater the unmet need to address a large variety of infectious diseases prevailing in the country.

Concurrent DENV infection, Chikunguniya virus, Zika virus infections are also recorded in a small proportion of cases.

In earlier outbreak more childhood cases were found then adult cases detected this year, a proportion elderly patients were also affected who had other co-morbidity

The WHO dengue Guidelines was used to update the National Guidelines, Bangladesh Society of Medicine (BSM) developed a short version as well. The BSM having a nationwide network with specialists was entrusted to provide training to health care professionals within short time which was sucessful. ${ }^{5}$

A number of atypical characteristics not attributable to plasma leakage(increase vascular fragility and permeability), gastrointestinal problem 10-49\%, pleural effusion, neurological manifestations- encephalitis, encephalopathy, GBS, myocardititis were also reported.

It was not unexpected to have co- infection with influenza (season from April to September), and co-infection with enteric fever. Other differential diagnosis needs to be considered during the outbreak. During outbreak only $20 \%$ were positive with dengue, what are the other organisms are in circulation with dengue? Unfortunately we do not have a capacity to diagnose cases of acute febrile illness nor such a broad range of point of contact user friendly diagnostic tests are available.

There was a continued steroid controversy for management of severe cases with a mixed opinion among physicians, quickly BSM planned for conducting an RCT.

Despite a large evidence generated through dengue research- issue of vaccine- to whom, and when are still not solved.
A new arena of sexual transmission of dengue- recent report from Europe from a returning traveler from Brazil is to be further confirmed.

We have to learn how to live with dengue due to country wide spread of infections. In a future introduction of new/different sero type of dengue virus in the population infected this year will be at risk of having secondary infection with a potential risk of severe dengue.

A vector control strategy according to the Global Vector Control Strategy involving the local Government Rural Development (LGRD), community, increasing the capacity with adequate vector biologists is a pre requisite for control of dengue. ${ }^{6}$

Till better diagnostics are available which is an urgent gap to mitigate and improved management without specific drug is not going to solve the problemand thus individual/self care and community engagement is crucially important.

Bangladesh Health Sector Programe is committed to provide quality care. For addressing diseases like dengue all of Government approaches, whole of society approaches, health in all policies should be adopted as we committed in the UN High Level Political Declaration. ${ }^{7}$

\section{Abul Faiz}

Professor of Medicine (Retired)

Former Director General of Health Services, \& Dev Care Foundation, Dhaka, Bangladesh,

E-mail: drmafaiz@gmail.com

\section{References:}

1. Yunus EB, Banu D, Talukder KR, Chowdhury MJH, Bangali A M and Montanari R M (2002). Seroepidemiological Study of Dengue/Dengue Haemorrhagic Fever in a Metropolitan Hospital in Bangladesh. Dengue Bulletin. Val.: 26: 1-6.

2. Faiz M A, Maude R J, Rahman M, Karim MJ, Zaman SI, White LJ, Aguas R (2014). Investigation into possible causes of upsurge of malaria in Bangladesh in 2014. Unpublished report. National strategic plan for malaria elimination: A path to the phased elimination of malaria from Bangladesh: February 2017, DGHS.

3. DGHS (17 Dec 2019). Dengue Status Report. https:// www.dghs.gov.bd/images/docs/Notice/2019/dengue/ Dengue_20191201.pdf

4. Mamun M A, Misti JM, Griffiths MD, Gozal D (2019). The dengue epidemic in Bangladesh: risk factors and actionable items. Lancet, Vol: 394: 2149-2150

5. DGHS \& WHO (2018). National Guideline for Clinical Management of Dengue Syndrome. $4^{\text {th }}$ Edition.

6. WHO (2017). Global Vector Control Response 20172030. Political Declaration of the High-level Meeting on Universal Health Coverage "Universal Health Coverage: Moving Together to Build a Healthier World". https:// www.un.org/pga/73/wp-content/uploads/sites/53/ 2019/05/UHC-Political-Declaration-zero-draft.pdf 